\title{
Phase behaviour of additive binary mixtures in the limit of infinite asymmetry
}

\author{
Yuri Martínez-Ratón and José A. Cuesta \\ Grupo Interdisciplinar de Sistemas Complicados (GISC), Departamento de Matemáticas, Escuela Politécnica Superior, \\ Universidad Carlos III de Madrid, c/ Butarque, 15, 28911 - Leganés, Madrid, Spain
}

\begin{abstract}
We provide an exact mapping between the density functional of a binary mixture and that of the effective one-component fluid in the limit of infinite asymmetry. The fluid of parallel hard cubes is thus mapped onto that of parallel adhesive hard cubes. Its phase behaviour reveals that demixing of a very asymmetric mixture can only occur between a solvent-rich fluid and a permeated large particle solid or between two large particle solids with different packing fractions. Comparing with hard spheres mixtures we conclude that the phase behaviour of very asymmetric hard-particle mixtures can be determined from that of the large component interacting via an adhesive-like potential.
\end{abstract}

PACS numbers: 61.20.Gy, 64.75.+g, 82.70.Dd

Since Biben and Hansen showed [1] that a binary mixture of hard spheres (HS) in Rogers-Young approximation has a spinodal instability for diameter ratios smaller than 0.2 , it is generally accepted that a demixing transition occurs in this fluid if the asymmetry between the two component sizes is large enough. This fact has been confirmed with other theories [2], simulations [3], and experiments in colloidal suspensions [- The question is no more whether such a mixture demixes but which is its phase behaviour. Some approximate theories [5] suggest that the phase rich in large spheres is a crystal instead of a fluid, in agreement with what is observed in the experiments 泊. The depletion potential between the large spheres due to the small ones has been determined both perturbatively 6] and in simulations [7]. If the interaction of the small spheres is replaced by this potential the resulting effective fluid can be simulated 7 [9]. In the unstable region of the binary mixture large clusters of depleted spheres are formed, which evolve very slowly [7]. The phase behavior of this effective fluid for diameter ratios smaller than 0.1 [8,9] shows a large coexistence region between a diluted fluid and a highly packed solid, and even an isostructural solid-solid transition, if the small spheres packing fraction and the diameter ratio are low enough.

The picture emerging from these studies is that the phase behaviour of a very asymmetric binary mixture can be understood in terms of the phase behaviour of a fluid of HS interacting via a depletion potential. This potential has a very narrow (essentially the diameter of the small spheres) and deep well [7]6], and this type of potential have been proven to have no liquid phase [10, 11]: there is only fluid-solid coexistence, or - if it is narrower and deeper - in addition there may appear an isostructural solid-solid transition.

In this letter we will explore this connection between the phase behaviour of an additive binary mixture and that of the effective depleted one-component fluid by explicitly showing that the Helmholtz free-energy functional of the mixture reduces to that of the corresponding one-component adhesive fluid in the limit of infinite asymmetry. That in this limit the solvent induces an ad- hesive interaction between the large particles is already a known result 12, 13]; but we are going a step further by mapping the functional of the mixture onto that of the corresponding adhesive fluid, and hence their respective phase behaviours.

The procedure is as follows. As usual in these systems we fix the chemical potential of the small particles, $\mu_{S}$ (semigrand ensemble), and therefore the system is described by the functional

$$
\Upsilon\left(\mu_{S},\left[\rho_{L}\right]\right)=F\left[\rho_{L}, \rho_{S}\right]-\mu_{S} \int \rho_{S}, \quad \mu_{S}=\frac{\delta F}{\delta \rho_{S}(\mathbf{r})},
$$

$\rho_{L(S)}$ being the density profile of the large (small) particles, and $F$ the Helmholtz free-energy functional. The equilibrium equation for $\rho_{S}$ in (1) is usually a nonlinear equation involving both densities; the infinite asymmetry limit then allows to explicitly obtain $\rho_{S}$ as an expansion with terms depending on $\rho_{L}$. This operation transforms (11) into an effective Helmholtz free-energy functional for the depleted large-component fluid (up to some divergent terms which do not affect the phase behaviour), with the fugacity of the solvent playing the role of an inverse temperature.

The direct correlation function (DCF) of the effective fluid is defined as minus the second functional derivative of the excess part of (11) with respect to $\rho_{L}$. It is straightforward to see that this leads to the following relationship with the components of the DCF of the mixture, written in Fourier space:

$$
\widehat{C}_{\text {eff }}(k)=\widehat{C}_{L L}(k)+\frac{\rho_{S} \widehat{C}_{L S}(k)^{2}}{1-\rho_{S} \widehat{C}_{S S}(k)} .
$$

In terms of the structure factor this expression is equivalent to assuming $S_{\text {eff }}(k)=S_{L L}(k)$. This is precisely the procedure used in [13] to map a binary HS mixture onto an adhesive HS fluid in the limit $\epsilon \equiv \sigma_{S} / \sigma_{L} \rightarrow 0, \sigma_{L(S)}$ denoting the diameter of the large (small) spheres.

To proceed with the functional mapping we have described above we need a free-energy functional able to deal with strong inhomogeneities (the solvent can be regarded as a fluid confined in between the large particles). 
Fundamental measure functionals are perfect candidates 14 17 both because of their ability to reduce dimensionality [15,17] and because they are formulated directly for mixtures. On the other hand, for the sake of simplicity we have chosen to apply this procedure to a binary mixture of parallel hard cubes (PHC). This fluid has also been shown to have a spinodal instability both in simulations on a lattice [18] and in continuum space [16], thus its phase behaviour can be expected to be qualitatively similar to that of HS. The procedure applied to the latter, however, has a higher level of complexity both because of the more complicated shape of the functionals [15] and because the weaker depletion in this fluid requires to expand to a higher order in $\epsilon$. There is nothing fundamental, though, which prevents its application to HS, and we are currently working along this line.

In order to determine the appropriate scaling of the solvent packing fraction $\eta_{S}$ we first take the $\epsilon \rightarrow 0$ limit in (2) forcing it to produce a finite nontrivial result. The right choice is $\eta_{S}=O(\epsilon)$; thus, if we define $\eta_{S}=\epsilon \xi$, the resulting DCF will be $\left(\sigma_{L}=1\right.$ in what follows) $C_{\text {eff }}=C_{\mathrm{PHC}}+C_{\mathrm{ad}}$, where

$$
C_{\mathrm{ad}}(\mathbf{r})=\frac{z}{2(1-\eta)}\left\{\delta_{\text {cont }}(\mathbf{r})+y S(\mathbf{r})+6 y^{2} V(\mathbf{r})\right\}
$$

$C_{\mathrm{PHC}}(\mathbf{r})$ is the DCF of a one-component $\mathrm{PHC}$ fluid 16, 17. of packing fraction $\eta=\eta_{L}$ (actually the total packing fraction in the limiting fluid); $z \equiv \epsilon^{2} \exp \left(\beta \mu^{\mathrm{ex}}\right)=$ $\xi /(1-\eta)$ is the "renormalized" excess (over ideal) fugacity of the solvent; $y \equiv \eta /(1-\eta)$; and

$$
\begin{aligned}
\delta_{\text {cont }}(\mathbf{r})= & \delta(1-|x|) L(y) L(z)+\delta(1-|y|) L(x) L(z) \\
& +\delta(1-|z|) L(x) L(y), \\
S(\mathbf{r})= & \Theta(1-|x|) L(y) L(z)+\Theta(1-|y|) L(x) L(z) \\
& +\Theta(1-|z|) L(x) L(y), \\
V(\mathbf{r})= & L(x) L(y) L(z),
\end{aligned}
$$

with $L(u) \equiv(1-|u|) \Theta(1-|u|)$ and $\Theta(v)=1$ if $v \geq 0$ and $=0$ otherwise.

Apart from corrections proportional to the overlap surface (5) and volume (6), at larger densities, the lowest density correction (4) is a delta on the contact surfacealso proportional to the actual contact surface. This has its origin in the effective adhesive potential on the surfaces of the cubes induced by the solvent, and it turns out to be a natural extension of that for HS 13,19 . We will thus refer to this fluid as the parallel adhesive hard cube (PAHC) fluid.

Once we know the scaling of the density we can determine the free energy with the procedure described above. The fundamental measure functional of a mixture of PHC is given by [17] $\beta F=\int d \mathbf{r}\left\{\Phi_{\mathrm{id}}(\mathbf{r})+\Phi_{\mathrm{ex}}(\mathbf{r})\right\}$, where

$$
\begin{aligned}
& \Phi_{\mathrm{id}}(\mathbf{r})=\sum_{i} \rho_{i}(\mathbf{r})\left(\log \left[\mathcal{V}_{i} \rho_{i}(\mathbf{r})\right]-1\right) \\
& \Phi_{\mathrm{ex}}(\mathbf{r})=-n_{0} \ln \left(1-n_{3}\right)+\frac{\mathbf{n}_{1} \cdot \mathbf{n}_{2}}{1-n_{3}}+\frac{n_{2, x} n_{2, y} n_{2, z}}{\left(1-n_{3}\right)^{2}}
\end{aligned}
$$

$\mathcal{V}_{i}$ being the thermal volume of species $i, \rho_{i}(\mathbf{r})$ its local number density, and $n_{0}, \mathbf{n}_{1}, \mathbf{n}_{2}, n_{3}$ are a set of "fundamental" weighted densities generically defined as the convolution $n_{\alpha}=\sum_{i} \rho_{i} * \omega_{i}^{(\alpha)}$, where $\omega_{i}^{(0)} \equiv \delta_{i}^{x} \delta_{i}^{y} \delta_{i}^{z}, \mathbf{w}_{i}^{(1)} \equiv\left(\theta_{i}^{x} \delta_{i}^{y} \delta_{i}^{z}, \delta_{i}^{x} \theta_{i}^{y} \delta_{i}^{z}, \delta_{i}^{x} \delta_{i}^{y} \theta_{i}^{z}\right), \mathbf{w}_{i}^{(2)} \equiv$ $\left(\delta_{i}^{x} \theta_{i}^{y} \theta_{i}^{z}, \theta_{i}^{x} \delta_{i}^{y} \theta_{i}^{z}, \theta_{i}^{x} \theta_{i}^{y} \delta_{i}^{z}\right), \omega_{i}^{(3)} \equiv \theta_{i}^{x} \theta_{i}^{y} \theta_{i}^{z}$, with $\theta_{i}^{u}=$ $\Theta\left(\sigma_{i} / 2-|u|\right)$, and $\delta_{i}^{u}=(1 / 2) \delta\left(\sigma_{i} / 2-|u|\right)$.

According to (1) the equilibrium scaled density of the solvent $\xi(\mathbf{r}) \equiv \epsilon^{2} \rho_{S}(\mathbf{r})$ is given by

$$
\log \xi(\mathbf{r})=\beta \mu_{S}^{\mathrm{ex}}+\ln \epsilon^{2}-\sum_{\alpha} \frac{\partial \Phi_{\mathrm{ex}}}{\partial n_{\alpha}} * \omega_{S}^{(\alpha)}(\mathbf{r}) .
$$

If we now take into account that, for any function $f(\mathbf{r})$, $f * \omega_{S}^{(\alpha)}=\epsilon^{\alpha} f+O\left(\epsilon^{\alpha+2}\right)$, for $\alpha=3$ or any vector component of $\alpha=2,1$, and $f * \omega_{S}^{(0)}=f+\left(\epsilon^{2} / 8\right) \nabla^{2} f+O\left(\epsilon^{4}\right)$, then $\xi(\mathbf{r})=z\left[1-\bar{n}_{3}(\mathbf{r})\right]+\epsilon \xi_{1}(\mathbf{r})+\epsilon^{2} \xi_{2}(\mathbf{r})+O\left(\epsilon^{3}\right)$ is the $\epsilon$-expansion of $\xi$ in terms of the $\bar{n}_{\alpha}$ and $z$ (for the sake of brevity we omit the expressions of $\xi_{1}$ and $\xi_{2}$ ). By overline we are denoting weighted densities of only the large component.

We can now insert the expansion of $\xi$ into the definition of $\Upsilon$ [Eq. (11)] and expand the whole functional in powers of $\epsilon$. This leads to

$$
\begin{aligned}
\Upsilon\left(\mu_{S},\left[\rho_{L}\right]\right) & =-\Pi_{0}(\epsilon) V+\mu_{0}(\epsilon) N+F_{\mathrm{PAHC}}+O(\epsilon), \\
\beta F_{\mathrm{PAHC}} & =\int d \mathbf{r}\left\{\bar{\Phi}_{\mathrm{id}}+\bar{\Phi}_{\mathrm{ex}}+\Phi_{\mathrm{ad}}\right\}
\end{aligned}
$$

The term $-\Pi_{0} V+\mu_{0} N$ diverges with $\epsilon \rightarrow 0$; nevertheless it is irrelevant for the phase behaviour because it simply adds $\Pi_{0}$ to the pressure and $\mu_{0}$ to the chemical potential of the large particles. These are two divergent constants which just cancel out in the equilibrium equations. The functions $\bar{\Phi}_{\mathrm{id}}$ and $\bar{\Phi}_{\mathrm{ex}}$ are given by (7) and (8) but only for the large component, and $\Phi_{\mathrm{ad}}$ is given by

$$
\Phi_{\mathrm{ad}}=\frac{z}{8} \frac{\left|\nabla \bar{n}_{3}\right|^{2}-4 \overline{\mathbf{n}}_{2} \cdot \overline{\mathbf{n}}_{2}}{1-\bar{n}_{3}} .
$$

A few things are worth noticing here. First of all we can see that out of this procedure a new weighted density has come up - namely $\nabla \bar{n}_{3}$ - which was missing at the beginning. Secondly, the second functional deriva-

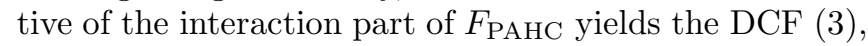
and this provides a consistency test. Finally, $\Phi_{\mathrm{ad}}$ is a negative contribution - it arises from an attractive interaction, as it can be easily realized particularizing for the uniform fluid (it can also be checked that this term is negative for any density profile).

The equation of state of the PAHC uniform fluid, in terms of $y=\eta /(1-\eta)$, turns out to be

$$
\beta P=y+3(1-z / 2) y^{2}+2 y^{3} .
$$

As in the case of the adhesive hard spheres (AHS) fluid [19 this equation has a van der Waals loop, and hence 
yields a gas-liquid phase transition (see Fig. 1) with a critical point at $z_{c}=2(1+\sqrt{2 / 3}) \approx 3.63, \eta_{c}=$ $(1+\sqrt{6})^{-1} \approx 0.29$

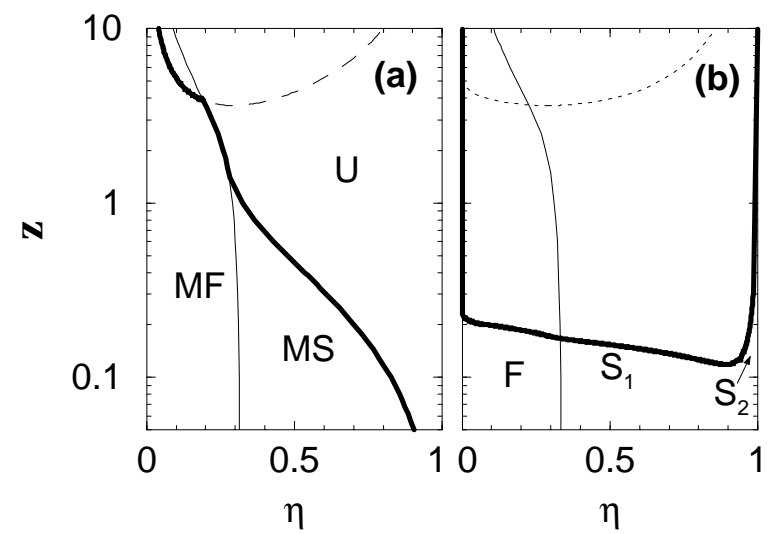

FIG. 1. Solvent fugacity, $z$, vs. solute packing fraction, $\eta$, of the infinitely asymmetric binary mixture of PHC, both without (a) and with (b) polydispersity $(\Delta \sigma / \sigma=4.5 \%$ ). (a) The thick solid line separates the unstable region (U) from the metastable one; the thin one marks the (continuous) transition from a metastable fluid (MF) to a metastable solid (MS); the dashed one is the fluid-fluid spinodal. (b) The thick solid line marks the fluid-solid or solid-solid coexistence; the thin one marks again the (continuous) fluid-solid transition below the coexistence region; the dotted line is the metastable fluid-fluid coexistence.

Allowing for inhomogeneous phases, however, changes dramatically the phase behaviour. As usual in density functional theories we determine the solid free energy by writing $\rho_{L}(\mathbf{r})$ as a sum of gaussians centered at the nodes of a (simple cubic) lattice, leaving their width as a variational parameter. We also account for vacancies by multiplying by an occupancy probability $\nu$, which acts as a second variational parameter. This occupancy can be expressed in terms of the lattice parameter, $d$, as $\nu=\eta d^{3}$; hence $d$ can be treated as an alternative variational parameter - subject to the constraint $d \geq 1$. First thing we can see is that the fluid-solid transition is continuous - therefore we actually obtain it directly from the DCF (3) by a standard bifurcation analysisand that it crosses the fluid-fluid coexistence at a packing fraction $\eta<\eta_{c}$. Hence the fluid-fluid transition is preempted by freezing.

But having $d$ as a variational parameter reveals that any of these phases is metastable. The reason is that at any value of $z, \Phi_{\mathrm{ad}} \rightarrow-\infty$ as $d \rightarrow 1$. Thus the only thermodynamically stable phases this system possesses are a closed-packed solid coexisting with a zero-density fluid. This is not surprising if one compares with the phase behaviour of adhesive hard spheres (AHS). Computer simulations of a square-well fluid with well-range going to zero [10] reveal that the AHS fluid also has the same phase behaviour. This singularity was already noticed by Stell [20], who proved that the partition function of the AHS fluid diverges for a system of 12 or more particles (12 is the coordination number of a fcc lattice). It simply expresses the fact that at any density and adhesiveness (solvent fugacity in our case) the system collapses into a close packed solid lattice.

Nevertheless, a more detailed exploration of the phase diagram shows that the fluid or the normal solid are sometimes local minima of the free-energy functional, and thus they are metastable phases, mostly separated from the close-packed solid by large free-energy barriers. The limit of mechanical stability of these metastable phases is shown in Fig. 1(a). It can be seen that the larger $z$ the larger the density range of metastability.

Thus the situation one will typically find in the PAHC fluid is the following. At large values of $z$ the fluid will quickly collapse into the close-packed configuration. At small values of $z$, however, the fluid will be trapped in metastable phases for very long times; upon increasing $\eta$ the fluid will be seen to undergo a continuous fluidsolid transition at $\eta \approx 0.3$, and it can remain solid for a very long period of time; if we move at still higher $\eta$ the system will eventually collapse, but as it may take long for the whole system to do it there may be an apparent expanded solid-collapsed solid coexistence. This picture is a caricature of the typical phase diagram of a fluid exhibiting an isostructural solid-solid transition [10,11].

A similar behaviour has been observed for the AHS fluid [21]: the phase diagram reveals a freezing transition in spite that the free energy becomes concave beyond a certain packing fraction (depending on the adhesiveness). The latter was interpreted in 21] as a percolation transition; however it is simply a sign of the collapse singularity, which did not show up because the density of vacancies was fixed to 0 (and hence the lattice parameter is forced to be $d=\eta^{-1 / 3}>1$; we observe the same behaviour in our system when we fix $\nu=1$ ). The reported phase diagram is thus metastable (compare the qualitative agreement with the one shown in Fig. 1(a) for the PAHC fluid).

The collapse of the adhesive potential can be removed by adding a small amount of polydispersity 20,22]. To see what the phase diagram looks like we have introduced a small amount of size polydispersity in the large cubes. In the fundamental measure formalism this simply amounts to replacing the weights $\omega_{L}^{(\alpha)}$ by their averages $\widetilde{\omega}_{L}^{(\alpha)}$ over the chosen size distribution. We have made the simplest computational choice for the latter: we have replaced the large cubes by parallelepipeds where each of their edge-lengths is chosen random and independently from a Gaussian distribution of mean 1 and deviation $\Delta \sigma$. It turns out that this choice transforms the former weights into "smoothed" versions of them, and that this removes the divergence of $\Phi_{\text {ad }}$ no matter how small $\Delta \sigma$ be. Besides, this choice leaves the free energy of the uniform fluid unchanged.

The results are plotted in Fig. 1(b). It can be seen that now we really have the typical phase diagram of a fluid 
exhibiting an isostructural solid-solid transition-except that for this system the fluid-solid transition is continuous. For large values of $z$ we have a very diluted fluid and a very dense solid separated by a large coexistence region. As we decrease $z$ the coexisting fluid eventually becomes denser until it reaches the density at which freezing occurs. From then on and down to a critical value, $z_{s}$, coexistence is between an expanded and a dense solid with the same crystal structure.

The results we have obtained can be reinterpreted in terms of the original very asymmetric binary mixture. The fluid phase would correspond to the stable mixture, and the solid phases to the solid of large particles permeated by a fluid of small particles. According to the PAHC phase diagram - either mono or polydisperse - no fluidfluid phase separation will ever occur for very asymmetric binary mixtures of $\mathrm{PHC}$; instead, at large solvent fugacity (large solvent density) there will be fluid-solid phase separation, and at smaller solvent fugacity the phase separation will take place after the large particles have crystallized.

Notice that the existence of this fluid-solid phase separation largely increases the region of the phase diagram where the mixture is unstable. This explains why recent simulations on very asymmetric binary mixtures of $\mathrm{PHC}$ [3] report the mixture to be unstable at packing fractions of the large component much smaller than those predicted by fluid-fluid phase separation [16] (notice that, according to the authors, the nature of the coexisting phases cannot be discerned in their simulation).

The same kind of phase behaviour has been very recently reported to occur in simulations of binary mixtures of HS at finite (but small) diameter ratios using the depletion potential [8] (they also show a solid-solid transition in a certain range of solvent fugacities). We can see then that this phase behaviour can be inferred from that of the AHS fluid [21. This result is of great importance because it establishes a connection between the phase behaviour of mixtures and that of the solvent fluid interacting via an adhesive-like potential [10,11, thus opening a new route to study demixing in hard-particle fluids. This remarkable enhancement of the slow divergence of the pair correlation function of two large bodies at contact, induced by depletion 12, is probably due to the fact that the confinement of the solvent in between the solute particles induces strong inhomogeneities in the former [see Eq. (9)] an hence largely increase depletion.

It is a pleasure to thank Daan Frenkel and Pedro Tarazona for illuminating discussions. This work is supported by project no. PB96-0119 from the Dirección General de Enseñanza Superior (Spain).
[1] T. Biben and J.-P. Hansen, Phys. Rev. Lett. 66, 2215 (1991).

[2] H. N. W. Lekkerkerker and A. Stroobants, Physica A 195, 387 (1993); Y. Rosenfeld, Phys. Rev. Lett. 72, 3831 (1994). T. Coussaert and M. Baus, Phys. Rev. Lett. 79, 1881 (1997); erratum: 80, 4832 (1998).

[3] A. Buhot and W. Krauth, Phys. Rev. Lett. 80, 3787 (1998).

[4] A. D. Dinsmore, A. G. Yodh, and D. J. Pine, Phys. Rev. E 52, 4045 (1995); U. Steiner, A. Meller, and J. Stavans, Phys. Rev. Lett. 74, 4750 (1995); A. Imhof and J. K. G. Dhont, Phys. Rev. Lett. 75, 1662 (1995).

[5] W. C. K. Poon and P. B. Warren, Europhys. Lett. 28, 513 (1994); C. Caccamo and G. Pellicane, Physica A 235. 149 (1997).

[6] Y. Mao, M. E. Cates, and H. N. W. Lekkerkerker, Physica A 222, 10 (1995).

[7] T. Biben, P. Bladon, and D. Frenkel, J. Phys.: Condens. Matter 8, 10799 (1996).

[8] N. García-Almarza and E. Enciso in Proceedings of the VIII Spanish Meeting on Statistical Physics FISES '97, p. 159, J. A. Cuesta and A. Sánchez, eds. (Editorial del CIEMAT, Madrid, 1998).

[9] M. Dijkstra, R. van Roij, and R. Evans, submitted to Phys. Rev. Lett. (1998).

[10] P. Bolhuis and D. Frenkel, Phys. Rev. Lett. 72, 2211 (1994); P. Bolhuis, M. Haagen, and D. Frenkel, Phys. Rev. E 50, 4880 (1994).

[11] C. F. Tejero, A. Daanoun, H. N. W. Lekkerkerker, and M. Baus, Phys. Rev. Lett. 73, 752 (1994); Phys. Rev. E 51, 558 (1995).

[12] T. Biben and J.-P. Hansen, Europhys. Lett. 12, 347 (1990).

[13] Y. Heno and C. Regnaut, J. Chem. Phys. 95, 9204 (1991).

[14] Y. Rosenfeld, Phys. Rev. Lett. 63, 980 (1989); see also Y. Rosenfeld, J. Phys.: Cond. Matter 8, 9289 (1996), and references therein.

[15] Y. Rosenfeld, M. Schmidt, H. Löwen, and P. Tarazona, J. Phys.: Cond. Matter 8, L577 (1996); Phys. Rev. E 55, 4245 (1997); P. Tarazona and Y. Rosenfeld, Phys. Rev. E 55, R4873 (1997).

[16] J. A. Cuesta, Phys. Rev. Lett. 76, 3742 (1996).

[17] J. A. Cuesta and Y. Martínez-Ratón, Phys. Rev. Lett. 78, 3681 (1997); J. Chem. Phys. 107, 6379 (1997).

[18] M. Dijkstra and D. Frenkel, Phys. Rev. Lett. 72, 298 (1994).

[19] R. J. Baxter, J. Chem. Phys. 49, 2770 (1968).

[20] G. Stell, J. Stat. Phys. 63, 1203 (1991).

[21] C. F. Tejero and M. Baus, Phys. Rev. E 48, 3793 (1993).

[22] D. Frenkel, private communication. 